\title{
Using Visualization Process Graphs to Improve Visualization Exploration
}

\author{
T. J. Jankun-Kelly \\ Department of Computer Science and Engineering \\ James Worth Bagley College of Engineering \\ Mississippi State University, MS 39762, USA \\ tjk@acm.org
}

\begin{abstract}
Visualization exploration is an iterative process of setting parameters, rendering, and evaluating results. This process can be recorded and analyzed in order to make visualization exploration more efficient and more effective. This work describes methods for visualizing the visualization process using new visualization process graphs; several visualization process relations are introduced to construct these graphs. These methods were used to analyze and improve a network routing visualization, and the results of this analysis are presented. Through this analysis, redundant exploration was quickly identified and eliminated.
\end{abstract}

\section{Introduction}

During the visualization process, a user iteratively explores a very large space of visualization parameters in order to discover results of interest. The search of this space can be costly - especially for expensive visualization techniques. It is vital that visualization systems be designed to streamline the exploration process, but support for this sort of optimization is not common. To understand and improve visualization exploration, a user's session must be recorded and analyzed. Unnecessary and expensive re-exploration could then be identified, suggesting ways to improve the visualization interface. Visualization process graphs, depictions of different characteristics of the user's exploration, can be used to perform this analysis, leading to improved systems. This form of provenance analytics is chiefly aimed at system designers, though there are potential uses by users as well.

This work describes new methods for extracting visual representations of the visualization process. Visualization sessions are themselves visualized using the process graphs introduced here. The formalism for constructing these graphs - visualization process relations - can also be used to directly analyze aspects of the visualization sessions. As a motivating example, a case study of how visualization graphs were used to improve a network visualization tool (the OASCBrowser [1]) is discussed. The OASCBrowser looks a changes of autonomous systems (ASes) via colored lines connecting ASes to IP addresses; clusters of these indicate anomolies. The graphs were used as a "visual profiler" to detect cycles and similar redundancies. This example illustrates how the process graphs can be used to make visualization more efficient. 


\section{Background}

Both scientific and information visualization can be described as an iterative process of data-filtering, visual mapping, and rendering with user interaction. Several approaches have been taken to present the history of the iterative exploration to the user. Where present, most visualization history depictions are linear graphs of next-previous relations (such as the Zoom Graphs in Polaris [2]). A more sophisticated history mechanism can be found in GRASPARC [3. GRASPARC presents a tree of branching parameter settings in a problem solving environment which includes visualization. The tree is used to step back to any previous simulation setting and branch the exploration. Finally, some novel visualization user interfaces present the visualization result provenance to the user, either as a depiction of the entire visual parameter space 4], a tabular projection of that space [5], a parallel coordinates depiction of the space [6], or an interactive graph of visualization derivations [7. Additional work has extended the depiction to include changes in the visualization's construction [8]. Recent work has also informally evaluated how to better measure the effectiveness of the visualization process 9,10 .

The previous works focused mainly on showing the history of the visualization or problem solving processes. However, for analyzing the visualization process, history alone is not sufficient. Often, there are relationships between the results and other elements of the visualization session which are vital to its understanding. The visual database exploration (VDE) work by Lee [11,12 has identified these elements for database exploration; Lee's work encapsulates multi-result relationships based upon database structural metadata. The VDE Model also uses graphs to display process information for database exploration; lessons from the VDE work are applied here. For visualization exploration, the P-Set Model of visualization exploration 13 was developed. The P-Set Model formalizes the visualization process and discusses relationships between results and the parameters which generate them; the details of these relationships are provided in the next section. The current work extends the P-Set Model by expanding the two process graphs previously discussed (the history and derivation graphs) and introducing two new process graphs (difference and containment graphs). In addition, a set of metrics and heuristics to evaluate these graphs is discussed. Taken together, the graphs and measures provide a framework for improving visualization tools such as the OASCBrowser system.

\subsection{The P-Set Model for Visualization Exploration}

Visualization process graphs depict links between different results in the session. These links are dependent upon the underlying model describing the visualization process. While the graphs discussed here could be distilled from other models of visualization exploration, the P-Set Model is used for the purposes of this work. 


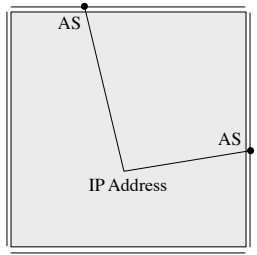

(a) OASC Layout

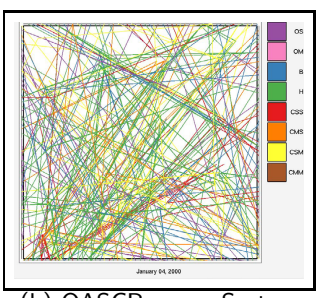

(b) OASCBrowser System

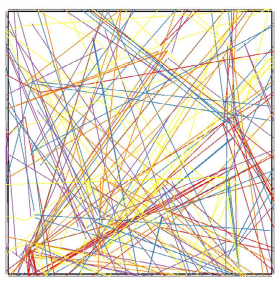

(c) Normal Events

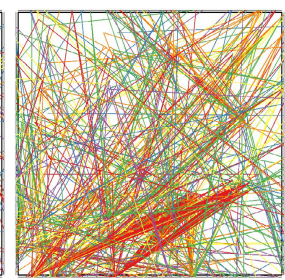

(d) Anomalous Events

Fig. 1. The initial Origin AS Change (OASC) Event visualization system, the OASCBrowser 1]. The ASes initiating an event (aligned around the square) are connected to the affected IP addresses within the square. Color indicates change type. Normal activity appears as a random collection of lines; anomalies in routing are detected by browsing through dates and active events types looking for correlated lines.

The P-Set Model of visualization exploration formalizes the iterative visualization cycle by describing a user's interaction with a visualization system. During such interaction, a user manipulates parameter values to form a parameter set (a $p$-set); a p-set is a collection of parameter values of different types (such as the date and active event parameters used by the OASCBrowser). Created p-sets are used to generate new results. A p-set, combined with a visualization transform (the operation which creates results), uniquely identifies a result; a result can be recreated given a p-set and transform. By recording how p-sets are derived from previous p-sets, the P-Set Model captures the salient details of the exploration process.

For each result generated during visualization exploration, four items are stored: a timestamp, parameter derivation information, p-set derivation information, and result derivation information. This four-tuple is known as a derivation. The timestamp indicates when the derivation was performed; it is possible for multiple results to be generated during the same timestamp as a consequence of a single user interaction. The parameter and p-set derivations describe which previous parameters and p-sets were used to create the new parameters and p-sets. Finally, the created results are identified by the p-sets and transforms used in their creation; the p-sets used must have been created via the parameter and p-set derivations. In this manner, each explored result is encoded by the model. Each of the four elements in a derivation will be used to form different visualization process graphs.

\section{Relations and Graphs for Visualization Analysis}

Visualization process graphs encode different relationships between results, parameters, and p-sets explored during a visualization session. These relationships are based upon different properties of these elements - when they were generated, what parameters they share, etc. In this section, four visualization process relations are introduced that are in turn used to define the process graphs. 

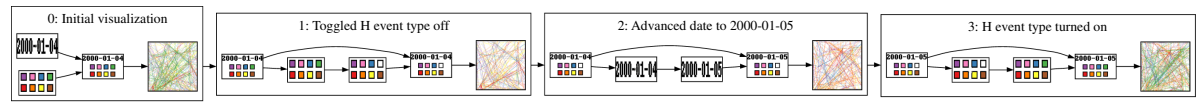

(a) History
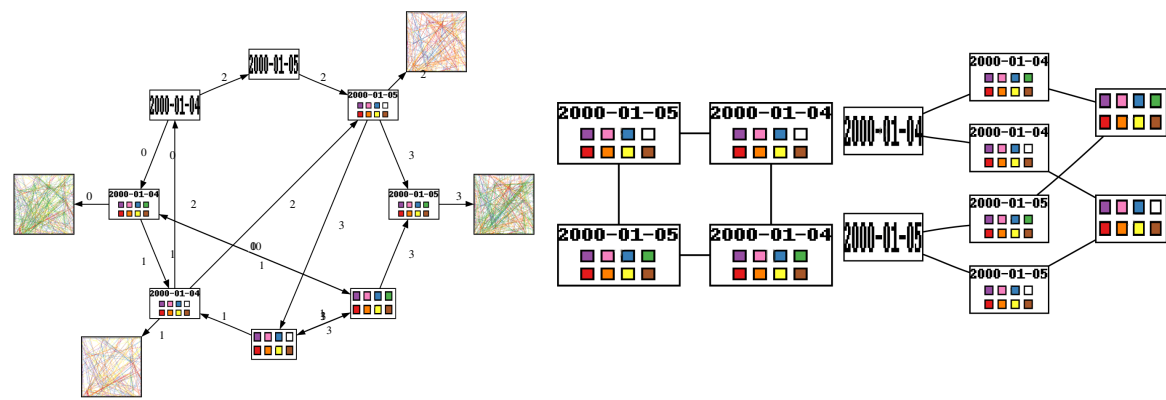

(b) Derivation

(c) Difference

(d) Containment

Fig. 2. Visualization process graphs for a OASCBrowser session. There are two parameters types in the graphs: dates and displayed OASC events. In the example, one event type was turned off, the next date's data was loaded, and finally all event types were toggled on again.

\subsection{Visualization Process Relations}

Four classes of visualization relations are used in this work: history, derivation, difference, and containment. Each relation is a boolean function over two elements in a visualization session; the function is true if the corresponding relation holds. Most of these operations relate parameters, p-sets, or results. Derivations, however, are vital to most of these relations, determining if a relation holds or not.

History Relation. The history relation imposes a linear temporal ordering upon the derivations in a session. This follows relation holds between two derivations if the latter derivation immediately follows the former (i.e., their timestamps differ by one). Since a derivation encapsulates several sets of parameter, p-sets, and results, this relation also imposes an ordering on these elements. The follows relation will be used to compose history information with derivation information from the derives relation.

Derivation Relations. In an individual P-Set Model derivation, four things occur. First, parameters are taken from existing p-sets to be modified. These input parameters are manipulated by the user to create output parameters. These output parameters are then applied to an input p-set from a previous result to create an output p-set. Finally, this output p-set is applied to a visualization transform to generate a result image. Each of these stages imposes a relationship between the two entities - i.e., the input parameters derive the output parameters. Thus, there are five derives relations: P-sets derive input parameters, input parameters derive output parameters, output parameters derive output p-sets, 
input p-sets derive output p-sets, and output p-sets derive results. It is important to note that it is possible for an element that was derived from another element to derive that self-same element - such cyclic derivations are indicative of redundant exploration. Thus, cycles in a chain of derivations will be used to identify ways to improve a visualization system.

Difference Relations. P-sets are the cornerstone element of the P-set Model. $\mathrm{P}$-sets are formed by direct user interaction and are the genesis of visualization results. Thus, a measure of the depth of the exploration is the depth of the visualization parameter space spanned by the p-sets. The differs relations measures the amount of difference between two p-sets, and thus indirectly captures the breadth of exploration. The differs relation used in this work is differs-by-one; two p-sets differ by one if they share the same parameter types but differ in only one value (e.g., only a colormap differs).

Containment Relations. The final set of relations are the contains relations. This relation is the normal mathematical containment relationship between an item and a set. In this context, the relation holds between a parameter and a p-set (if the parameter is contained within the p-set) or a parameter and a result (if the parameter is contained within the p-set used to generate that result). Similar to the differs relation, the containment relation measures the depth of exploration. If a single parameter value is shared among many p-sets, then further exploration could be facilitated by using a different parameter value for those same p-sets.

Using Visualization Process Relationships. Each set of relationships highlights different aspects of the visualization process. Though parameter derivation information is not present in the history relation, it gives a clear sense of the flow of time during the visualization process. The derivation relations provide a sense of parent-child relationships; this relationship could be combined with the history relation or used without. Combined, the derives and follows relations could identify how a result or p-set was first created. Alone, the derives relation details how that result/p-set was used in subsequent exploration. Finally, the difference and containment relations give a sense of the depth of exploration during the process. Shallow spanning trees of graphs using this relation signify a visualization process that did not deeply search the space of parameter values while deep or broad spanning trees could suggest lack of focus. In fact, using these relations to build graphs is a powerful method for performing visualization session analysis. This idea will be explored in-depth next.

\subsection{Visualization Process Graphs}

Each visualization process relation has a corresponding visualization process graph; these graphs visually summarize the relations. The graphs introduced here are similar in purpose and properties to the graphs of the VDE model. However, these graphs are tailored to visualization exploration. In addition, the difference and containment graphs have no analog in the VDE work. To illustrate 
the process graphs, Figure 2 will be used as an example; for an explanation of the OASCBrowser system used in the example, refer to Figure 1 and Section 4.

History Process Graph. The history process graph (Figure 2a) provides a visual overview of the temporal ordering of results. Each element in the sequence is a derivation that was generated by the user in a single operation. These elements are drawn from the domain and range of the follows relation. History can be displayed graphically using vertices representing the derivation and directed edges representing time.

The history graph is a line connecting each derivation; in interfaces that expose session information, this is the type of graph usually displayed. To be more informative, derivations components within a single time-step can be included (such as in Figure 2a). The parameters, p-sets, and results within a derivation are ordered based upon the derives relation: Inputs are sources while results are sinks in the derivation subgraphs. Thus, each derivation is depicted as a sequence of parameter, p-set, and result derivations. For example, node 2 in the figure clearly shows how the date from the previous result's p-set was changed from January 4, 2000 to January 5, 2000 to create a new p-set and result for the following day's events. Note, though the same p-set or parameter may be used in different derivations, each derivation's subgraph possesses a unique node for its p-set or parameter instance. This prevents edges between nodes belonging to different derivations (i.e., different time-steps); such relations are the purview of the derivation graph discussed next.

Like the OASCBrowser system, most visualization systems have derivations with only one input p-set, one input and output parameter, and one generated result. This occurs because most of these interfaces cannot manipulate more than one parameter, cannot utilize more than the immediately preceding p-set, and cannot generate more than one result at a time. A system which relaxes these constraints, such as the Image Graph [7, would have correspondingly more complex derivation information such as multiple input p-sets or created results.

Derivation Process Graph. The history process graph is insufficient for describing the relationships between results, parameters, and p-sets. Over the course of an exploration session, different parameters from the same p-set may be used to create multiple results. In addition, a result may be visited more than once. These relations are not explicitly present in the history graph. The derivation process graph captures this information (Figure 20).

A derivation process graph is constructed from three types of nodes: parameters, p-sets, and results. For each derivation, an edge exists between two nodes if the former derives the latter according the the derives relation. Unlike the history graph, there is only one node per parameter, p-set, or result. Thus, nodes may have multiple incoming or outgoing edges due to derivations involving that node at different time-steps. To disambiguate these edges, they are labeled with the corresponding derivation's timestamp.

The derivation graph succinctly summarizes the ancestor-descendent relations within the visualization session; paths in the graph correspond to sequences of 
derivations. Landmark parameters and p-sets are easily identified by possessing a large number of incoming and outgoing edges. In addition, redundant exploration is identified by p-sets nodes with multiple incident edges with distinct time-stamps. Such edges indicate that the p-set was visited by multiple user interactions, re-creating its associated result each time. Analysis of Figure $2 \mathrm{~b}$ shows that there was no redundant exploration (every p-set was explored only once) while one parameter was used twice (the parameter signifying that all OASC event types were displayed, lower left corner of Figure 2b).

Difference and Containment Process Graphs. The p-set difference graph and the p-set containment graph highlight the depth of exploration whereas the other graphs highlight the structure of the exploration. A difference process graph (Figure 2c) connects two p-sets if they vary by a certain number of parameter values; all difference graphs in this paper connect p-sets that differ by only one parameter. Each partition in these graphs represents similar results. The more clusters there are, the larger the explored parameter space. In Figure 2 , there are two partitions of the graph based upon parameter type - the top/bottom partition separate p-sets by which event types are displayed, while the left/right partition separates the dates explored. The number and size of such partitions are proportional to the size of the parameter space explored. Many, small partitions indicate a broad exploration of several different parameter types while few, large partitions correspond to a session with significant depth in one parameter.

Containment graphs (Figure 2d) depict how the explored p-sets are composed from the explored parameters: An edge exists between a parameter and a p-set if the parameter belongs to the p-set. The relative number of parameters of different types and p-sets are another indicator of the depth of exploration. Parameter types which dominate the exploration are easily spotted by how they outnumber the other parameter type nodes. If the number of p-sets dominates the graph, then the parameter space was highly explored. In Figure $2 \mathrm{~d}$, every valid combination of parameter values generated was explored during the session.

Using Visualization Process Graphs. Visualization process graphs serve two functions: They provide an overview of the visualization process and allow that process to be analyzed. The history and derivation graphs are most useful in the former application; the last three are vital for the latter. The history graph, augmented with derivation information, could be used as a browser over the visualization session; the Image Graph can be considered an implementation of a subset of this graph. Like the Image Graph, the history graph could be made interactive. This interaction would provide a sophisticated mechanism to build results from previous results. Such a system is beyond the scope of this discussion.

Several methods for analyzing visualization process graphs have already been introduced (i.e., looking at the number of incident edges to a p-set with differing time-stamps in the derivation graph). These methods can be formalized via visualization process metrics. Each metric is a function over a graph, node, or 
edge and summarizes properties of the graph; the properties relate to characteristics of the exploration. Previously, Lee [12] developed a set of metrics for the VDE model; we adapt some of them and introduce several new ones. The metrics introduced here are tailored to visualization exploration, and measure statistics relevant to such exploration:

- Importance Metric. For a given node in a derivation process graph, the important metric counts the degree of the node - the total number of incoming and outgoing edges. Nodes with a higher degree were derived by or derived multiple results in the exploration session, and thus were integral to that process. For the sample session, the p-sets which derived the second and third results have the most importance; however this difference is not significant (the next importance value is only one less than the maximum).

- Redundancy and Efficiency Metrics. For a given derivation process graph, the redundancy metric counts the number of incident edges to any p-set which have different time-stamps. This metric could be applied directly to p-sets to count the number of times the p-set was re-explored. Recall that psets, combined with visualization transform, uniquely identify a result; thus, a measure of the redundancy of one is a measure of the redundancy of the other.

Given a redundancy value, a measure of efficiency can be derived. The inefficiency of a session is the ratio of the redundant edges to the total number of p-set derivation edges in the derivation graph; the efficiency is one minus this number. For the sample session, the efficiency was $100 \%$ - no p-set was explored more than once.

- Depth Metric. The value of the depth metric is the maximum number of neighbors of any node in the p-set difference graph. This value is also the size of the largest cluster in the difference graph. Since each node in this cluster must differ by only one parameter type, the sub-space of the parameter space spanned by this type was well explored. For the sample session, the depth value was 2 parameter settings.

- Breadth and Coverage Metrics. The breadth of a visualization session is defined as the number of p-sets explored during the session-the more psets generated, the larger the explored visualization parameter space. A more meaningful measure is coverage; this is the number of p-sets explored over the possible number of valid p-sets in the session. This value can be calculated from the containment graph: The total number of valid p-sets for a single visualization transform is the product of the count of parameter values nodes for the transform's associated parameter types (e.g., if there are 3 dates and 2 selections of events, there are 6 possible valid p-sets); the total number of valid p-sets is the sum of the valid p-sets for each transform. For the sample session in Figure 2d, the coverage was 100\% since there were two parameters values for each of the two parameter types and four explored p-sets. While the coverage metric is useful for understanding the extent of the exploration, is not necessarily a measure of its effectiveness - not all combinations of parameter values are equally meaningful. 
These metrics provide heuristics to evaluate a visualization system. Assuming that the re-exploration of results is costly and should be avoided, systems should strive for a high efficiency (limiting redundancy). If a certain parameter has a high depth value, it suggests that a means to more quickly explore this sub-space of the visualization parameter space could be beneficial. While process graphs do not capture every characteristic of a visualization system (such as its runningtime or memory efficiency), they capture the essential properties of explorations using that system. The use of the graphs and their associated will be explored in more depth in the following section.

\section{Case Study: Improving the OASCBrowser}

The OASCBrowser [1] is a tool for visually detecting anomalies in internet routing information (Figure 1). The tool displays different types of changes to ownership of autonomous systems (ASes) - groups of hosts on the Internet. The different types of changes (called $O A S C$ events) are labeled with different colors. The colored lines connect ASes along the edge of a square to points within the square corresponding to the IP address affected by the change. The tool allows a user to browse through recorded dates with different types of AS changes highlighted. Anomalies are found by visually searching the dates for unusual patterns - normal behavior appears random while abnormal behavior appears as correlated lines.

The user can manipulate two different parameter settings for the OASCBrowser: The currently displayed date, and which OASC events to display. There are eight event types; the display of each can be individually toggled. The OASCBrowser does not provide a history mechanism - only the last result may be manipulated by the user. Thus, to compare a previous result, a user must recreate its parameter settings manually. As will be demonstrated, this leads to significant inefficiency during exploration.

\subsection{Example Session and Analysis}

Several visualization sessions were analyzed; all showed similar behavior. In an exemplar session, a range of OASC events between August 1st and August 22nd, 2000 were visualized. During this exploration, three sets of anomalies were discovered: A pair of correlated anomalies near August 1st, a major anomaly and its correction between the 14th and 17th, and another pair of correlated events on the 21st. In the session, 61 parameters and 76 p-sets and results were generated. Since there was only one visualization transform (the OASC Event visualization), there is a one-to-one correspondence between p-sets and results.

Figure 3 depicts the derivation, containment, and difference graphs for this session. For clarity, the derivation graph has been collapsed into a different (but equivalent) view of the session that shows only result derivations. Since there is a one-to-one correspondence between these two graphs (as there is only visualization type), the graphs are isomorphic. 

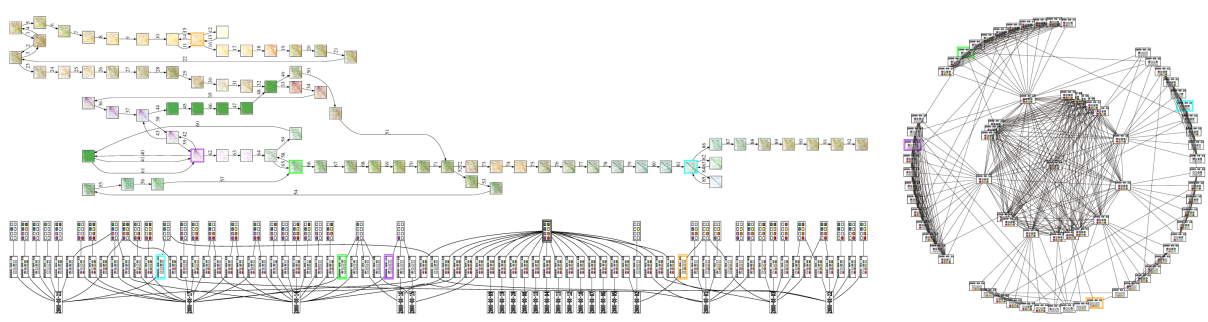

Fig. 3. Visualization process graphs for the original OASCBrowser viewing events during August 1-22, 2000. Top-left: Result derivation. Bottom-left: P-Set Containment. Right: P-set Difference.

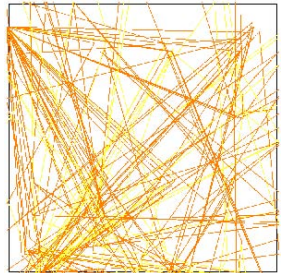

(a) August 1 (orange)

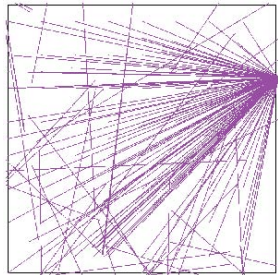

(b) August 14 (purple)

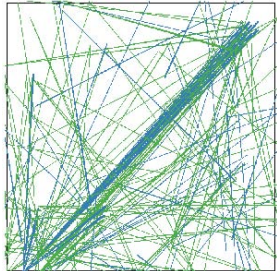

(c) August 21 (cyan)

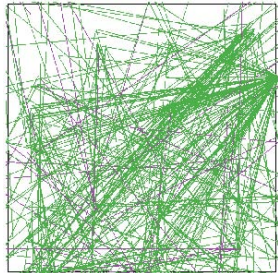

(d) August 17 (green)

Fig. 4. Landmark results in the example exploration session. Each is highlighted in the graphs in Figure 3 using the given color. The August 1st and 21st results show two correlated anomalies each; each pair of events is of different types. The August 14th result shows anomalous events that are later corrected by events on the 17 th.

Several observations can be made from the session's process graphs. First, there are several cycles in the exploration. The cycles occur in three portions of the derivation graph: The upper half (the August 1st anomalies), the lower left (the major anomaly between August 14th and 17th), and the lower right of the graph (the August 21st anomalies). In fact, the three most important results in the derivation graphs (according to the importance metric) correspond to these three anomalies (Figure $4 \mathrm{a}-\mathrm{c}$, outlined in orange, purple, and cyan respectively in Figure 3). For the August 1st and 21st anomalies, it is telling that each important result displays the two correlated events together; the derivation graph shows that these results were generated multiple times while the user toggled the two events on and off in order to isolate them.

The exploration of the August 14th anomaly is more complex. Upon initial inspection, the visualization is flooded with $\mathrm{H}$ (green) events. However, further analysis shows that the cause of these corrective events is one AS's OS (purple) events; these events correspond to the result highlighted in the figures. The exploration cycle here is complex for several reasons. First, there are the multiple anomalous events (the $\mathrm{H}$ and OS events) on the August 14th date; this causes the toggling seen before. However, there is a another set of anomalous H events three days later from the same AS (Figure 4d, highlighted in green in Figures 3); these are another set of corrective events. In the exploration session, the user compared the 14 th and 
17th events by stepping back and forward in time and changing which events were displayed. This caused the nested loops in the derivation graph.

As demonstrated, the given exploration session was inefficient: It has $70 \%$ efficiency according to our efficiency metric with 28 redundant derivations - over a quarter of the exploration was spent recreating previous results. From the difference graph, it can be confirmed that most of this redundant exploration was over one parameter type, the displayed events - most of the outer clusters represents toggling active events while keeping the date fixed. The center cluster represents p-sets stepping through time. The distribution of parameter values in the containment graph also supports the inefficient parameter exploration conclusion. The parameter corresponding to rendering all event types (bold border in the upper middle of the figure) covers $30 \%$ of the p-set nodes in the graph. Thus, $70 \%$ of the exploration consisted of setting the active event types to find the events of interest. Considering that only four dates of the 22 viewed had significant anomalies, this parameter exploring is unneeded. A more effective interface would reduce the need for excessive parameter searching.

\subsection{The Refined OASCBrowser}

From the previous analysis, it was concluded that the OASC event browsing interface was inefficient when drilling-down into an anomaly - too much redundant activity was spent toggling through the parameter values to find the event types of interest. The occlusion of the events was determined to be the cause of this toggling; analysts could not perceive specific events when exploring an anomaly. However, the ability to show all the event types simultaneously in one image was found useful to quickly spot when anomalies occur. Thus, a new interface was designed that showed both the overview visualization and visualizations for each of the event types concurrently (Figure [5, see Teoh [14] for details). By using multiple views, unneeded parameter generation is avoided.

Figure 5 depicts the process graphs for the exploration of the August 1st22nd, 2000 events using new the interface. The new interface does improve the exploration, though it does not remove all inefficiencies. The difference graph
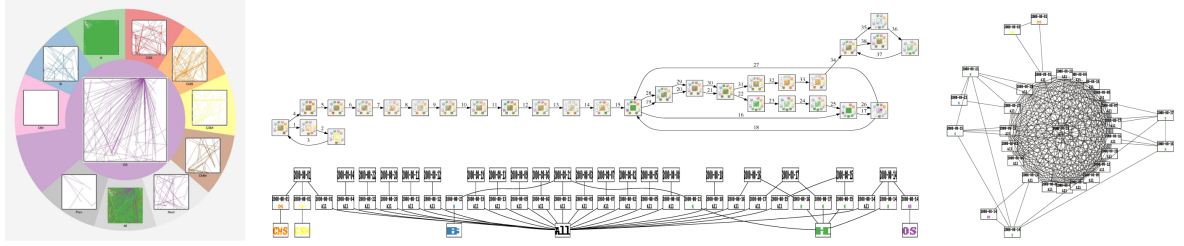

Fig. 5. The refined OASCBrowser interface (showing the anomalous events of August 14th, 2000) and corresponding process graphs for the exploration of August 1-22, 2000. The new interface displays the combined OASC event view in addition to a view for each individual event; events from the surrounding dates are also shown. This reduces the amount of redundant exploration compared to the original interface. Middle-Top: Result Derivation. Middle-Bottom: P-Set Containment. Right: P-Set Difference. 
shows that the exploration was more dense; the majority of the p-sets only changed their date parameter. Because the new interface allowed users to drilldown to individual events directly, more interactions were spent exploring the dates than the event types. The containment graph corroborates this finding; it shows that only $30 \%$ of the parameter exploration focused on individual anomaly types. Since the majority of dates did not have anomalies, this exploration is more in line with the actual data, unlike the original interface.

While the new interface does reduce the need for excessive parameter manipulation, it still suffers from inefficiency. For the given session, the overall efficiency is $56 \%$, less than that of the original interface. If the results exploring the $\mathrm{Au}-$ gust 14-17 events are excluded, the efficiency rises to $85 \%$, an improvement over the original browser. This signifies that for correlated anomalies on the same date (such as the August 1st and 21st anomalies), the new interface is more efficient - fewer derivations are needed to drill-down to individual events. However, the new OASCBrowser does not solve the problem of comparing anomalies from different dates; a user is still required to re-explore the intervening date's results. Further interface refinement could address this issue.

\section{Discussion}

Analyzing a user's process via the process graphs allowed us to improve the OASCBrowser. The graphs clearly identified cycles in exploration and reinforced some issues we had previously identified. It is important to note the the exact metrics are user dependent - each user will have slightly different patterns - so aggregate or holistic examination of the different sessions will be required. All users of the tool in our case study were familiar with it, so the question of how novice and expert sessions vary is still open.

The P-Set Model explicitly captures changes in parameters, not in other aspects of the visualization. For systems such as the OASCBrowser which are not modular (i.e., not extendable by adding new components or by changing the visualization pipeline), the $\mathrm{P}$-Set Model is adequate. For modular visualization environments, the P-Set Model can capture interactions with complete pipelines, but not explicitly capture changes within the pipeline. Extensions to capture pipeline changes are possible so long as the model is derivational-modifications to the more transaction-based approach used in VisTrails [8] is conceivable initial approach. In this sort of model, the visualization transform of a p-set would refer to complete transforms which would be built/modified by the transform model. Annotation of such changes (e.g., why the user performed an operation) is easily added by capturing it and using an RDF-like model [15] to extend the XML representation [13.

While the metrics presented are agnostic to the number of parameters used in the visualization, the graph-based representation may need to be condensed or collated in some fashion. This is especially true for the difference relation: More possible parameters will explode the number of possible nodes. An intelligent clustering scheme would elide individual nodes to display only groups of 
interest. For very long sessions, a similar graph summarization approach would be needed for the containment and derivation relations. Such condensation is especially important if the graphs are to be used by users directly; only more recent derivations will be typically of interest, and chains of non-branching derivations could be summarized.

\section{Conclusions}

The provenance of the visualization process is complex. To gain an understanding of visualization sessions - and perhaps a better understanding of the data originally visualized - this information needs to be visualized. Four graphs examining different parts of the visualization process were presented, and an in-depth example demonstrated the uses of this kind of analysis.

There are several potential applications for the work presented here. Visualizations of the visualization process give insight into the process and the interfaces used. The analysis of both OASCBrowser interfaces suggested areas of refinement, and a quantifiable improvement was observed for the refined interface. Similar analysis could be performed on other visualization systems. Other potential uses include communicating results and exploration sessions to collaborators, validation of sessions, and navigation of the visualization parameter space during exploration.

\subsection{Future Work}

As mentioned, one area of further study is the development of additional relations and metrics for visualization process analysis. Of interest is the identification and quantifying of the common patterns within process graphs. If quantified, a datamining system could then be used to automatically identify these patterns and call attention to them when displaying the process. This automation would assist in the understanding of the visualization process. Besides improving the visualization interface, this analysis could also be used to perform usability studies utilizing the metrics presented here.

Another avenue of research is the integration of visual analysis tools with visualization systems. During the visualization, the user could also be presented with visualization process graphs summarizing the exploration. This depiction could help in parameter space navigation and be used as a history tool to return to different results in a manner similar to the Image Graph.

\section{Acknowledgments}

The author would like to thank Kwan-Liu Ma and Soon Tee Teoh for their input and discussion and for access to the original OASC visualization tool. Melanie Tory feedback on an initial draft of this work are appreciated. GraphViz from AT\&T Research was used to generate the graph images. 


\section{References}

1. Teoh, S.T., Ma, K.L., Wu, F., Zhao, X.: Case study: Interactive visualization for internet security. In: Proc. of 13th IEEE Conference on Visualization (Vis 2002), pp. 505-508 (2002)

2. Stolte, C., Tang, D., Hanrahan, P.: Multiscale visualization using data cubes. IEEE Trans. on Visualization and Computer Graphics 9(2), 176-187 (2003)

3. Brodlie, K., Poon, A., Wright, H., Brankin, L., Banecki, G., Gay, A.: GRASPARCA problem solving environment integrating computation and visualization. In: Proc. of the 4th IEEE Conference on Visualization (Vis 1993), pp. 102-109 (1993)

4. Marks, J., Andalman, B., Beardsley, P.A., Freeman, W., Gibson, S., Hodgins, J., Kang, T., Mirtich, B., Pfister, H., Ruml, W., Ryall, K., Seims, J., Shieber, S.: Design Galleries: A general approach to setting parameters for computer graphics and animation. In: Proc. of ACM SIGGRAPH 1997, pp. 389-400 (1997)

5. Jankun-Kelly, T.J., Ma, K.L.: Visualization exploration and encapsulation via a spreadsheet-like interface. IEEE Trans. on Visualization and Computer Graphics $7(3), 275-287$ (2001)

6. Tory, M., Potts, S., Möller, T.: A parallel coordinates style interface for exploratory volume visualization. IEEE Trans. on Visualization and Computer Graphics 11(1), 71-80 (2005)

7. Ma, K.L.: Image Graphs-A novel approach to visual data exploration. In: Proc. of the 10th IEEE Conference on Visualization (Vis 1999), pp. 81-89, 513 (1999)

8. Bavoil, L., Callahan, S.P., Crossno, P.J., Freire, J., Scheidegger, C.E., Silva, C.T., Vo, H.T.: Vistrails: Enabling interactive multiple-view visualizations. In: Proc. of the 16th IEEE Conference on Visualization (Vis 2005), pp. 135-142 (2005)

9. Perer, A., Shneiderman, B.: Integrating statistics and visualization: case studies of gaining clarity during exploratory data analysis. In: Proc. of CHI 2008, pp. 265-274 (2008)

10. Shrinivasan, Y.B., van Wijk, J.J.: Supporting the analytical reasoning process in information visualization. In: Proc. of CHI 2008, pp. 1237-1246 (2008)

11. Lee, J.P., Grinstein, G.G.: An architecture for retaining and analyzing visual explorations of databases. In: Proc. of the 6th IEEE Conference on Visualization (Vis 1995), pp. 101-108 (1995)

12. Lee, J.P.: A Systems and Process Model for Data Exploration. PhD thesis, U. of Mass. Lowell (1998)

13. Jankun-Kelly, T.J., Ma, K.L., Gertz, M.: A model and framework for visualization exploration. IEEE Trans. on Visualization and Computer Graphics 13(2), 357-369 (2007)

14. Teoh, S.T., Jankun-Kelly, T.J., Ma, K.L., Wu, S.F.: Visual data analysis for detecting flaws and intruders in computer network systems. IEEE Comp. Graph. and Applications 24(5), 27-35 (2004)

15. Lassila, O., Swick, R.R.: Resource Description Framework (RDF) Model and Syntax Specification. Technical report, World Wide Web Consortium (1999), http://www.w3.org/TR/REC-rdf-syntax 